\title{
CHARACTERISTICS OF TEXTURE INDEX OF DAMAGED BUILDINGS USING TIME-SERIES HIGH-RESOLUTION OPTICAL SATELLITE IMAGES
}

\author{
M. Sonobe ${ }^{1}$ \\ ${ }^{1}$ Department of Civil Engineering College of Science and Technology, Nihon University, Japan \\ sonobe.masashi@nihon-u.ac.jp
}

KEY WORDS: Disaster, Earthquake, Damaged buildings, Texture analysis, dissimilarity

\begin{abstract}
:
A large-scale disaster has occurred due to the earthquake. In particular, $20 \%$ of the world's earthquakes with a magnitude of 6 or more occur near Japan. Damage analysis of buildings by image analysis have been effectively carried out using optical highresolution satellite images and aerial photograph with spatial resolution of about $2 \mathrm{~m}$ or less. In this study, the damaged buildings caused by large-scale and continuous earthquakes in Kumamoto, Japan that occurred in April 2016 was selected as a typical example of damaged buildings. For these earthquake event, the applicability of damage distribution of buildings and recovery/restoration status by texture analysis was examined. The applicability of the representative in the dissimilarity texture analysis methods GrayLevel Co-occurrence Matrix (GLCM) method by image interpretation in the case of a large number of collapsed and wrecked buildings in a wide area was assessed. These results suggest that dissimilarity was applicable to the extraction of damaged and removed buildings in the event of such an earthquake. In addition, the analysis results were appropriately evaluated by comparing the field survey results with the image interpretation results of the pan-sharpened image. From these results, we confirmed the effectiveness of texture analysis using time-series high-resolution satellite images in grasping the damaged buildings before and immediately after the disaster and in the restoration situation 1 year after the disaster.
\end{abstract}

\section{INTRODUCTION}

On the 2016, two large earthquakes occurred continuously on April 14 and April 16 in Kumamoto prefecture, Japan (GSI, 2017). In Mashiki town located near the epicenter due to this earthquake, 3000 buildings were completely destroyed and a massive building damage occurred (CAO, 2017). Immediately after the disaster, the damaged buildings were identified mainly through field surveys. Four years after the disaster, restoration and reconstruction work is still in progress to restore buildings and roads, water and sewage. In the case of such a large-scale damage, it is necessary to grasp the damage situation as soon as possible, and it is effective to collect damage information by satellite remote sensing. Continuous use of satellite information over the long term is expected to effectively restore the infrastructure and reconstruction of the community. Moreover, the obtained evaluation result is profitable spatial information for the disaster prevention in the future. Furthermore, the resolution of the satellite image has been increased, and Method of object-based and pixel-based texture for extracting detailed damage information of the building and grasping the damaged area have been studied (Hussain et al., 2011; Chen et al., 2018; Suzuki et al., 2016; Horlick et al., 1973; Miura et al., 2012; Huyck et al., 2005). In addition, cases of using UAV that can obtain high-resolution images have also been reported (Ghaffarian and Kerle, 2019). In general, many parameters are required for image segmentation and image classification by the object-based method. On the other hand, Texture-based analysis can be performed with relatively few parameters. The damage caused by the optical and SAR images observed immediately after the damage has been investigated and the damage of the building by the field survey is being investigated (Liu et al., 2017; NILIM and BRI 2017; Sumida et al., 2019). As a use of texture analysis for building damage in the 2016 Kumamoto earthquake, studies using aerial photographs and SAR satellite images are being conducted (Naito et al., 2018; Yamada et al.,
2017). However, long-term time series surveys before and after the disaster and several years after the disaster using texture analysis of satellite images have not been studied. In this study, the characteristics of the texture index were evaluated using high-resolution satellite images observed before and after the earthquake and 1 year after the earthquake.

\section{DATA}

\subsection{Satellite data}

QuickBird satellite data observed before the earthquake (March 24, 2004). QuickBird satellite sensor produces 4-band multispectral images and a panchromatic image with a resolution of $0.6 \times 0.6 \mathrm{~m}$ and $2.4 \times 2.4 \mathrm{~m}$, respectively. WorldView-3 satellite data observed after the earthquake (May 5, 2016) and 1 years after the earthquake (March 11, 2017). WorldView-3 satellite sensor produces 8-band multispectral images and a panchromatic image with a resolution of $0.3 \times 0.3$ $\mathrm{m}$ and $1.2 \times 1.2 \mathrm{~m}$, respectively. These three satellite image data have almost the same season (Figure 1).

\subsection{GIS data}

The building area was created by converting the perimeter lines of buildings issued by the GSI (The Geospatial Information Authority of Japan) to polygons. Information on the presence or absence of removal was added to the building polygons by image interpretation from the pan-sharpened image 1 year after the disaster. For the evaluation of the analysis results, the building damage survey results investigated by the Architectural Institute of Japan (AIJ) were used. 


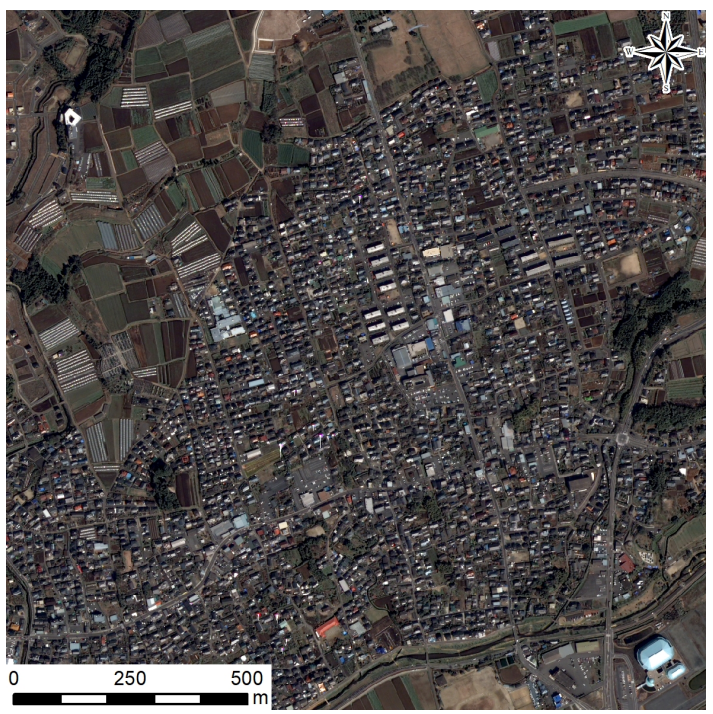

(a) Before disaster

(Acquisition date: March 24, 2017)

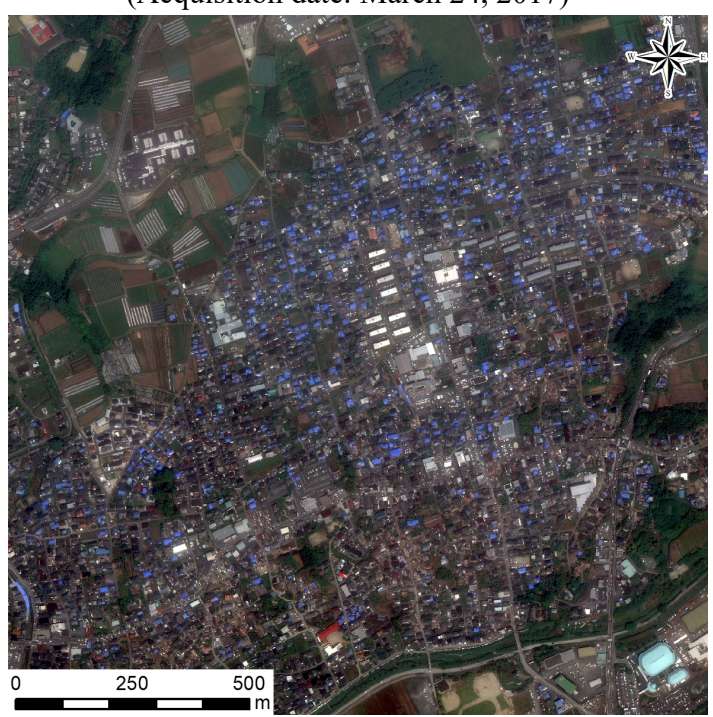

(b) Immediately after the disaster

(Acquisition date: May 5, 2016)

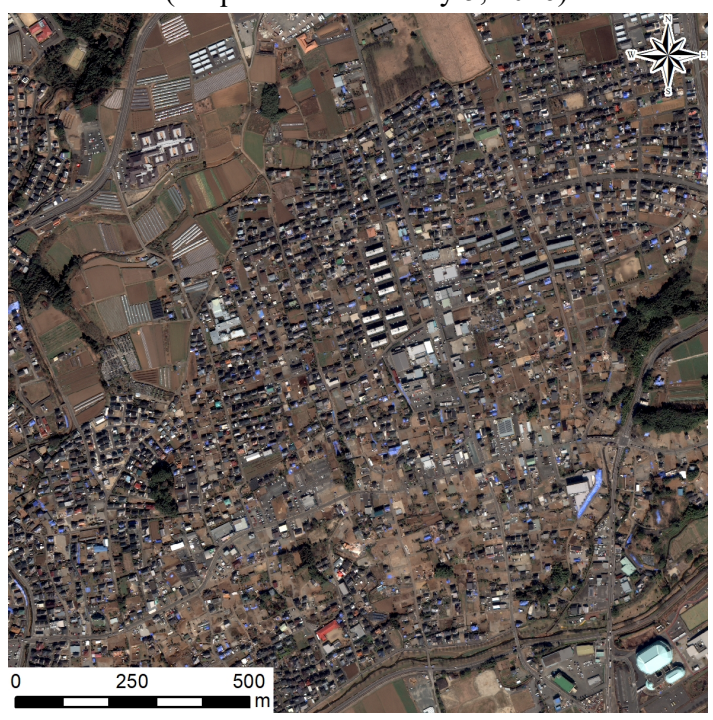

(c) After the disaster

(Acquisition date: March 11, 2017)

Figure 1. Satellite image in study area

\section{METHODS}

\subsection{Study area}

The study area was $1.5 \times 1.5(\mathrm{~km})$ in Mashiki town, which was severely damaged by the 2016 Kumamoto earthquake (Figure 2). There are about 3,300 building polygons, and the number of meshes in the building damage survey results is 329 . The Akitsu River flows from west to east on the south side of the study area, and the old river channel exists along the Akitsu River.

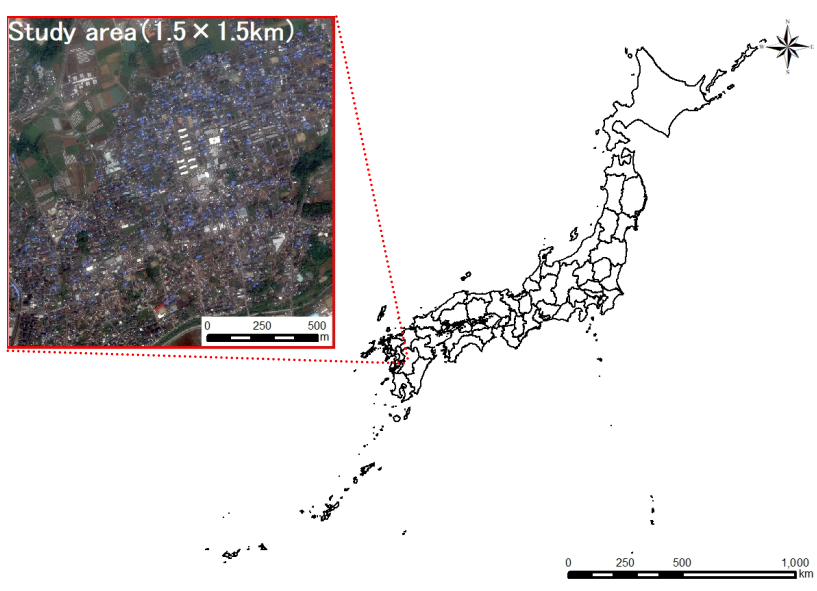

Figure 2. Study area

\subsection{Texture analysis}

First, the texture index was calculated using the co-occurrence matrix, and the distribution of building damage was evaluated. In this study, we used the dissimilarity as a texture index based on the Gray-Level Co-occurrence Matrix (GLCM). The dissimilarity has the characteristic that it becomes high at the place where the bright pixel and the dark pixel are adjacent (rubble etc.). In the damaged building, the rubble generated from the roof and the walls is scattered, so the bright and dark pixels are adjacent around the building area, and it is assumed that the texture is not uniform. In the restoration and reconstruction phase, it was presumed that the texture becomes uniform as the debris is removed. Therefore, the dissimilarity was adopted as the texture index based on the Gray-Level Cooccurrence Matrix (GLCM). The dissimilarity calculation formula is shown in (1).

$$
\text { dissimilarity }=\sum_{i=0}^{255} \sum_{j=0}^{255} P(i, j)|i-j|
$$

where $i$ is row number and $j$ is column number. $P(i, j)$ is probability value recorded for the cell $i, j$.

The band used was the red band after pan-sharpening. The pixel size was resampled to $0.6 \mathrm{~m}$. The frequency distribution of pixel values of usage data is different. For this reason, pixel values with cumulative frequency between $2 \%$ and $98 \%$ were used to convert to 8-bit images. The window size for calculation of the dissimilarity was set to $25 \times 25$ pixels. These processing methods were applied to three satellite images. The characteristics of building damage were grasped by image interpretation from the obtained time-series dissimilarity images. Also, statistics were calculated with building polygons containing information on the removal 1 year after the disaster. 


\subsection{Grasp building condition based on dissimilarity}

Next, in order to examine the grasp of the building condition, the damaged buildings immediately after the disaster and the restored buildings 1 year after the disaster were extracted using the dissimilarity. Note that damaged buildings were judged from the dissimilarity immediately after the disaster. By setting a threshold, damaged and undamaged buildings were classified. The set threshold value was the mean value of polygons that aggregated the dissimilarity immediately after the disaster. The classification results were compared with the building damage rate (destruction rate) of the building damage survey results. The building damage survey results is the value obtained by dividing the damage level of wreck, collapse, and collapse in the mesh area of about $57 \times 57 \mathrm{~m}$ by the total number of buildings in the mesh area. The building damage survey results was set based on Damage Grade (D1 D6) judged by the Architectural Institute of Japan (Okada and Takai, 1999). The wreck rate is the ratio of the number of buildings (destruction; D4) or more in the mesh area divided by the total number of buildings in the mesh area. The extraction of the buildings that were removed by the restoration and reconstruction work used the dissimilarity immediately after the disaster and 1 year after the disaster. The normalized value was calculated using the dissimilarity of the building polygons immediately after the disaster and 1 year after the disaster. The removed buildings

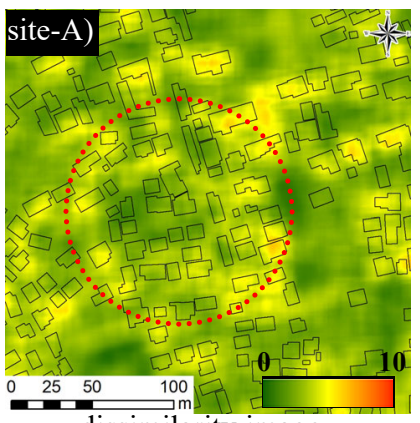

dissimilarity image

(a) Before disaster (Acquisitic

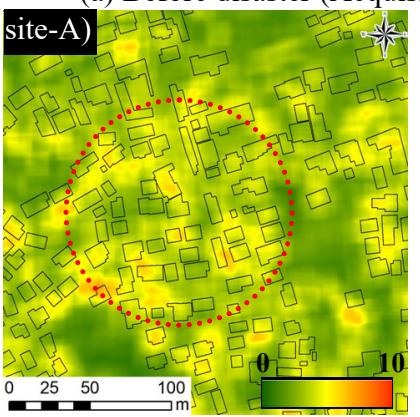

dissimilarity image

(b) Immediately after the disaster (Acquisition date: May 5, 2016)

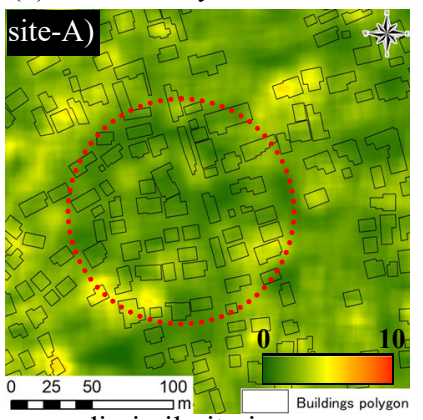

dissimilarity image

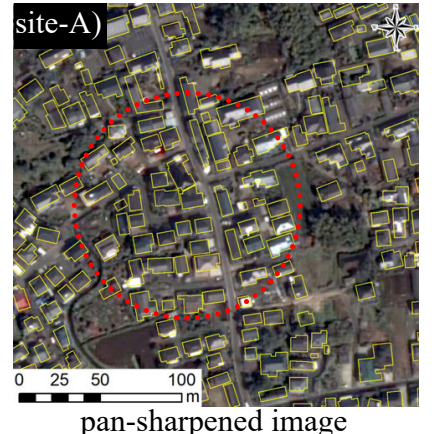

pan-sharpened imag

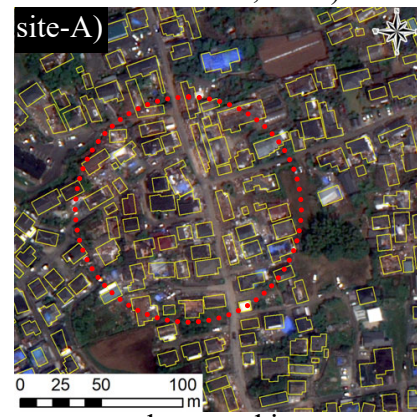

pan-sharpened image

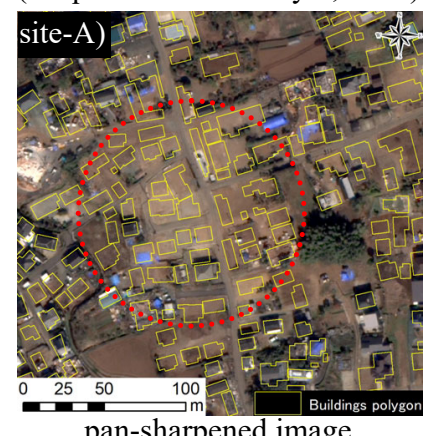

pan-sharpened image (c) After the disaster (Acquisition date: March 11, 2017) Figure 3. Comparison of dissimilarity in site-A

were classified by setting the threshold value to the mean value of the normalized values for each building polygon. (2) as follows:

$D I S S_{N O R M}=\left(D_{I S S_{2016}}-D_{I S S_{2017}}\right) /\left(D_{I S S_{2016}}+D_{I S S_{2017}}\right)$

where $D_{1 S S_{2016}}$ is the dissimilarity processed by WorldView-3 images observed on May 5, 2016. DISS 2017 is the dissimilarity processed by WorldView-3 images observed on March 11, 2017.

The analysis result was evaluated by comparing with the result of image interpretation. Accuracy verification was evaluated by extraction rate and justification rate. The extraction rate indicates the ratio of the removed building polygons extracted from the satellite image in the removed building polygons by image interpretation. The justification rate indicates the ratio of the building polygons removed by image interpretation among the extracted building polygons.

\section{RESULTS AND DISSCUSSION}

\subsection{Interpretation by the dissimilarity images}

Figures 3 and 4 show heterogeneity images and pan-sharpened images for three periods at two locations within the study area.

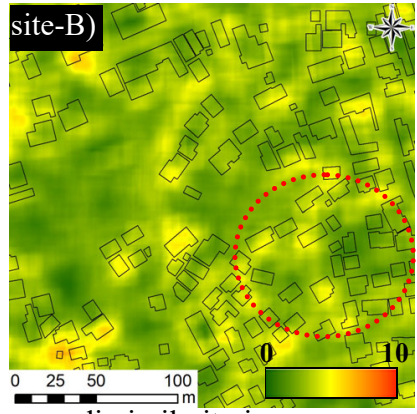

dissimilarity image

(a) Before disaster (Acquisition c

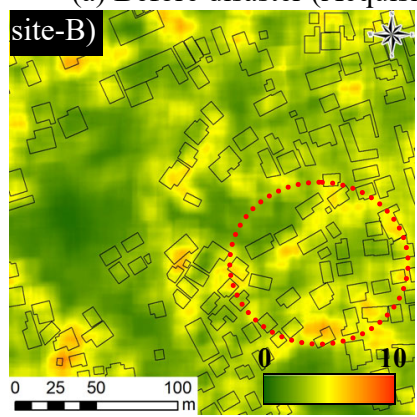

dissimilarity image

(b) Immediately after the disaster (Acquisition date: May 5, 2016)

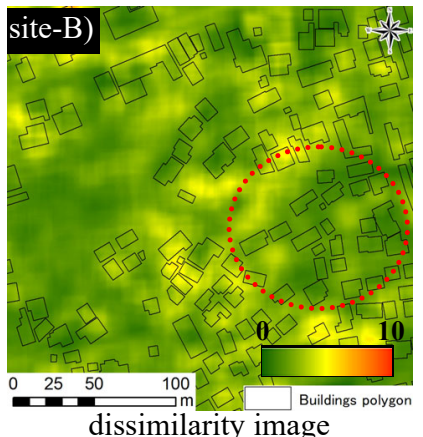

dissimilarity image

(c) After the disaster (Acquisition date: March 11, 20

Figure 4. Comparison of dissimilarity in site-B 
Immediately after the disaster, high values were observed around buildings where rubble was generated due to building damage. These were confirmed from the enlarged views before and immediately after the disaster. A high value is also distributed in the buildings that are being removed 1 year after the disaster. After the disaster, the situation became uniform due to the removal of rubble and land readjustment projects, and the tendency for the dissimilarity to decrease was confirmed. In addition, areas with continuous cars on roads and parking lots tended to have a high the dissimilarity. The building polygons near roads and parking lots are expected to increase in the dissimilarity due to the influence of vehicles. The blue tarp covering the roof immediately after the disaster was not affected by the use of the red band. It was possible to grasp the approximate distribution of the damage status of buildings and the restoration status from the dissimilarity images of the three periods obtained from the texture analysis.

\subsection{Change of the dissimilarity in the building polygons}

The mean value in the building polygons was calculated from the dissimilarity images of three periods. The results of tabulation are shown in Table 1 . The mean value was 4 to 4.5 , and the standard deviation was 1.1 to 1.4 . Moreover, in image interpretation, the dissimilarity tended to increase due to debris immediately after the disaster, but comparing the mean values before and immediately after the disaster, the values before the disaster were higher. It is considered that these are due to the long difference in satellite observation period before and after the disaster and to different satellites. Focusing on the mean value of the removed building polygons 1 year after the disaster, the dissimilarity tends to be lower than immediately after the disaster because many damaged buildings have become uniform. Similarly, the standard deviation of the removed building polygons 1 year after the disaster was confirmed to be lower 1 year after the disaster. On the other hand, it was confirmed that 1 year after the disaster, unremoved buildings that had been damaged had high values due to the increase in debris being removed.

\begin{tabular}{c|c|c|c|c|c|c}
\multirow{2}{*}{ Obsebation Date } & \multicolumn{3}{|c|}{ Mean } & \multicolumn{3}{c}{ Standard deviation } \\
\cline { 2 - 7 } & Not removal & Removal & All & Not removal & Removal & All \\
\hline March 23, 2004 & 4.40 & 4.58 & 4.45 & 1.14 & 1.09 & 1.13 \\
\hline May 5, 2016 & 3.92 & 4.22 & 4.01 & 1.16 & 1.08 & 1.13 \\
\hline March 11, 2017 & 4.83 & 3.38 & 4.40 & 1.27 & 0.95 & 1.36
\end{tabular}

Table 1 . The dissimilarity statistics

\subsection{Classification of damaged building polygons by the dissimilarity}

From the result of totaling the dissimilarity in the building polygons, it was not possible to confirm the tendency that the pixels became disordered before and after the disaster. Therefore, we examined the classification of damaged and undamaged buildings using the dissimilarity immediately after the disaster. Figure 5 shows the dissimilarity within the building polygons immediately after the disaster. It was confirmed that polygons with a high the dissimilarity tended to be distributed on the south side. From the enlarged view, it was found that the location where rubble was generated was highly heterogeneous due to the damage caused by the earthquake. Therefore, the

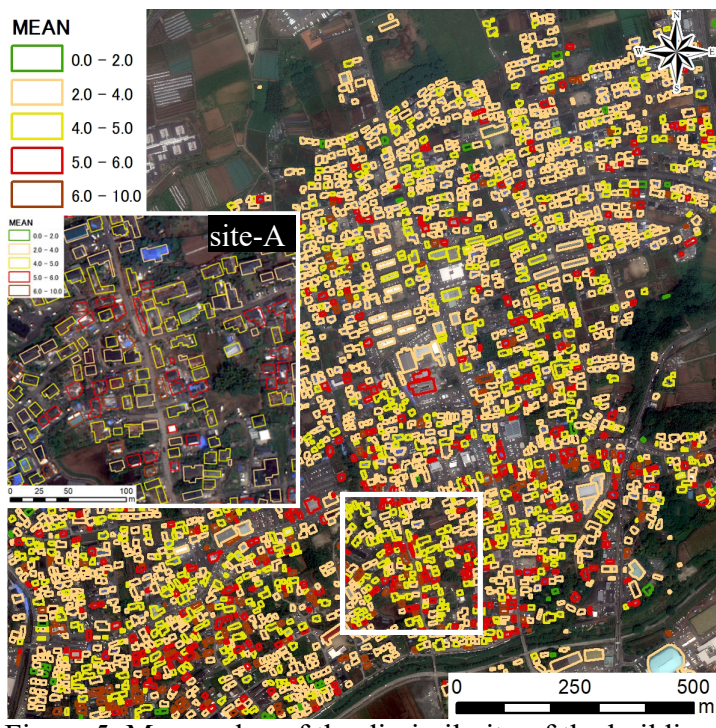

Figure 5. Mean value of the dissimilarity of the building polygons

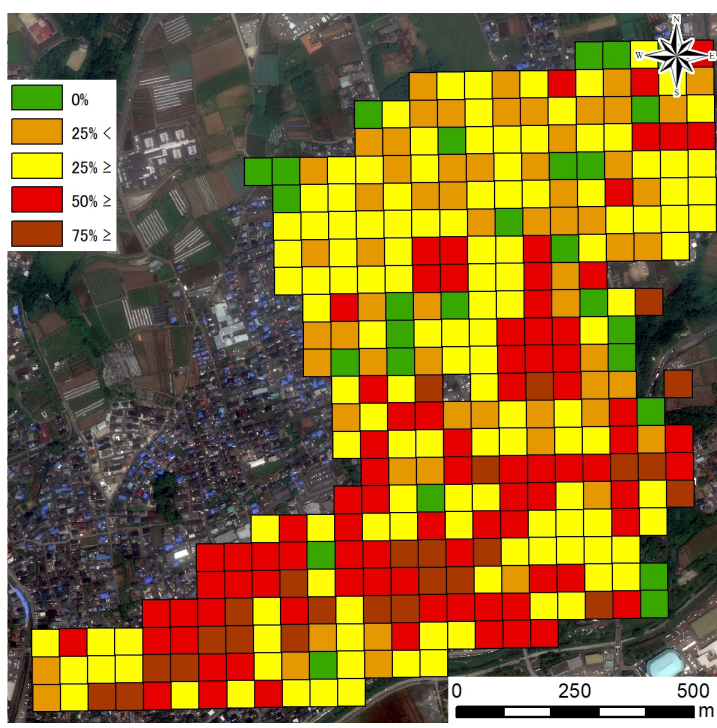

(a) The buildings damage rate with mesh calculated from the dissimilarity

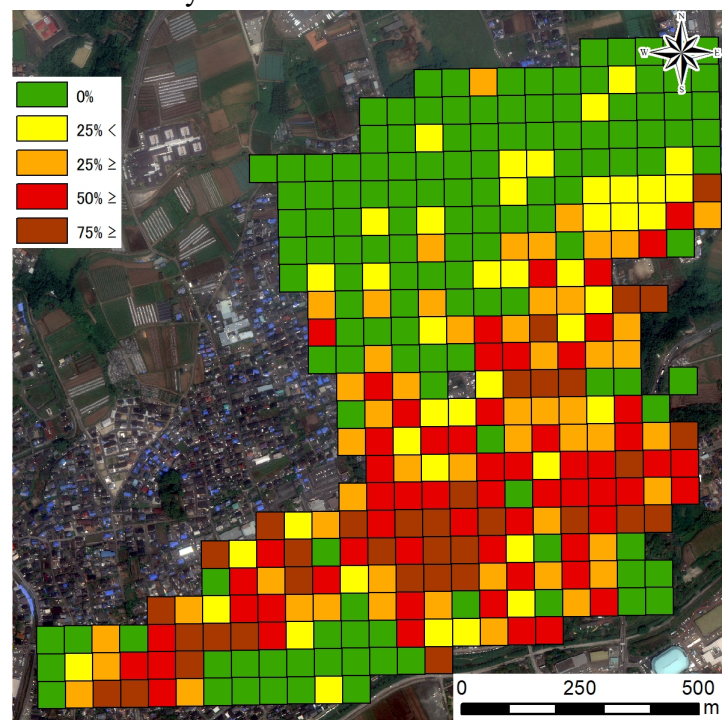

(b)The building damage survey (destruction rate) by AIJ Figure 6. Comparison of the destruction rate and the buildings damage rate using the dissimilarity 
mean value of the dissimilarity within the building polygons immediately after the disaster was set as the threshold value. In addition, the results classified into 5 levels are shown in Figure 6-(a) for comparison with the results of the building damage survey results. Figure 6-(b) shows the results of a comprehensive survey by the Architectural Institute of Japan (AIJ). There was a tendency for overestimation on the north side of the target area where damage was particularly small. Assuming use during a disaster, it is important to identify a location where damage is large during a disaster and take prompt rescue measures. Therefore, we compared meshes with a crushing rate of $50 \%$ or more between the building damage survey results and satellite analysis results. It was found from the building damage survey results that the damaged area was concentrated in the old river channel, and the mesh created from the satellite analysis results showed a similar distribution. The extraction rate was $73 \%$. The extraction rate indicates the ratio of buildings with $50 \%$ or more of damaged buildings in satellite image analysis for meshes with a crushing rate of $50 \%$ or more as a result of the building damage survey results. From these, it was shown that the distribution tendency of damaged buildings could be grasped from the dissimilarity obtained from high-resolution satellite images immediately after the disaster.

\subsection{Classification of removal building polygons by the dissimilarity}

As a survey of the restoration status, focused on the removal status of damaged buildings. Figure 7 shows the result of the judgment of the removed building polygons by image interpretation using the pan-sharpened image one year after the disaster. One-third of the building polygons had been removed in the study area within 1 year from the disaster. In addition, many of these are distributed on the south side where damage is concentrated. From Table 1, it was confirmed that the mean value and the standard deviation of the dissimilarity tended to decrease when comparing the removed building polygons immediately after the disaster and 1 year after the disaster. From these results, the polygon-based classification of removed buildings was based on the dissimilarity immediately after the disaster and 1 year after the disaster. Figure 8 shows the normalized values of the building polygons. In the case where the dissimilarity is reduced, the normalized value is 0 or more. As mentioned above, the dissimilarity of the Removed buildings tends to decrease as the soil is leveled. Therefore, it is estimated that the removed buildings tend to show a normalized value of 0 or more. It was found that 0 or more building polygons were distributed on the south side where the damaged buildings were concentrated. In addition, the normalized value was higher in the removed building compared to the image interpretation result. On the other hand, within the building polygons that has not been removed, 1 year after the disaster becomes a high value due to the increase in rubble being removed. Therefore, the removed buildings were classified using the mean value of the normalized values of polygons as a threshold. As a result of the classification, the extraction rate was $73 \%$, the justification rate was $63 \%$ (Table 2 ). The main misidentification factors were vehicles on roads and parking lots. In addition, it was speculated that the building could not be observed with debris from satellite images immediately after the disaster. Furthermore, it is considered necessary to consider the relationship between the types of buildings. From these results, it was possible to determine the existence of polygonbased removal using the dissimilarity. In addition, it was also possible to confirm the restoration status from the mesh created from the dissimilarity image 1 year after the disaster.

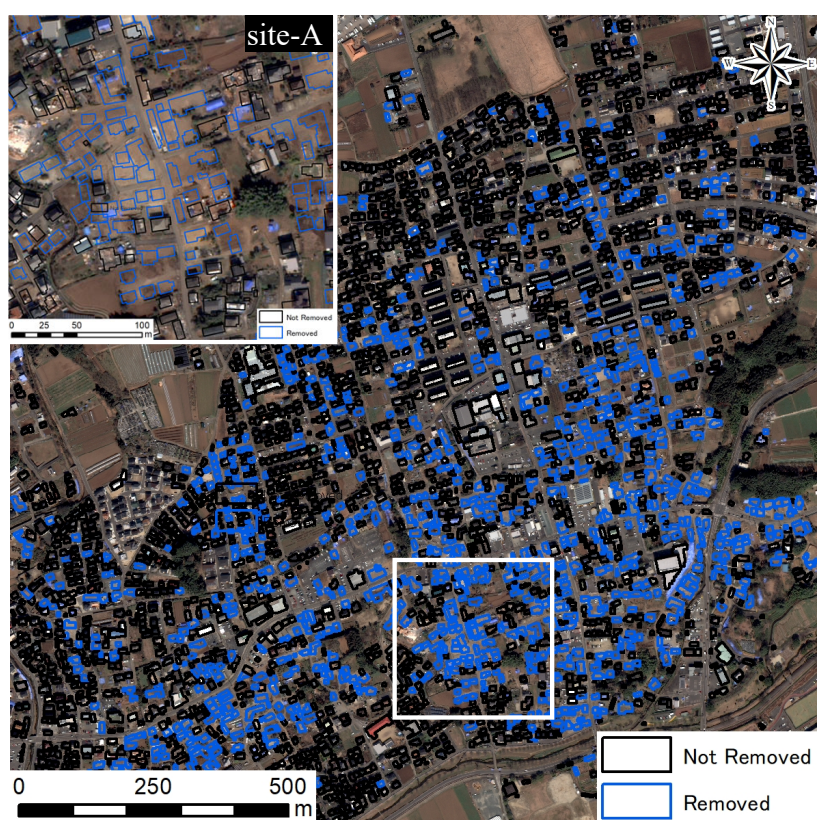

Figure 7. Extraction of removed building polygons by image interpretation

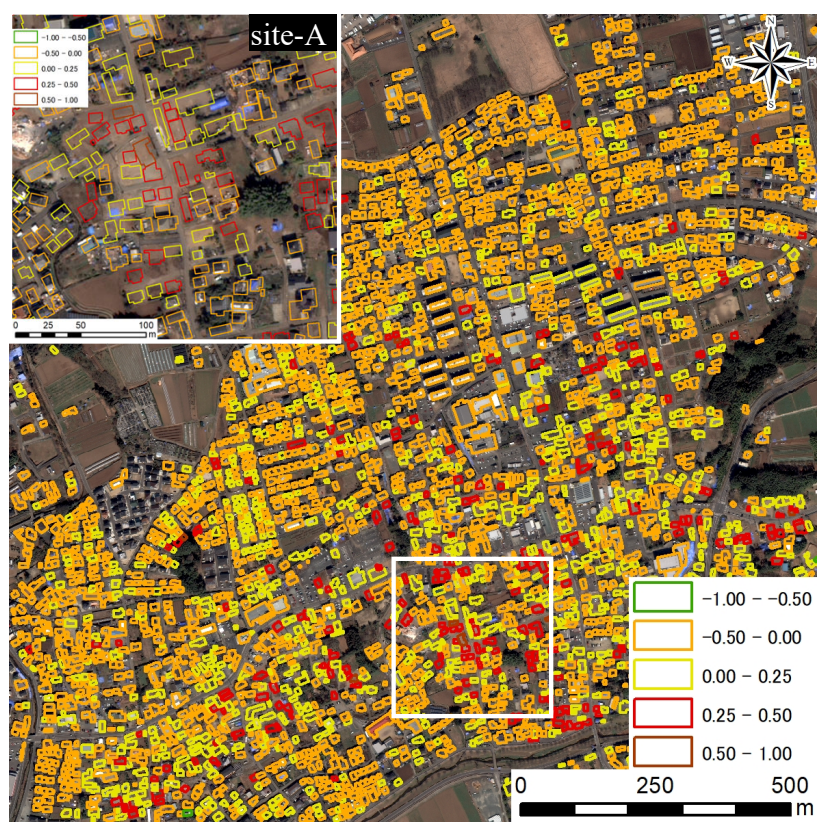

Figure 8. Extraction of removed building polygons by analysis

\begin{tabular}{c|c|c|c}
\multicolumn{2}{c|}{} & \multicolumn{2}{c}{ Extraction by satellite images } \\
\cline { 3 - 4 } \multicolumn{2}{c|}{} & Removal & Not Removal \\
\hline $\begin{array}{c}\text { Image } \\
\text { interpretation }\end{array}$ & Removal & (a) 713 & (b) 271 \\
\cline { 2 - 4 } & Not Removal & (c) 418 & (d) 1,906
\end{tabular}

Extraction rate $[\%]=a /(a+b): 73 \%$

Justification rate $[\%]=a /(a+c): 63 \%$

Table 2. Evaluation of classification accuracy 


\section{CONCLUSION}

In this study, using high resolution satellite observation data observed in time series before and after the disaster, for the 2016 Kumamoto earthquake, the texture index by the GrayLevel Co-occurrence Matrix (GLCM) is calculated, and examined the grasp of the state of the building from the distribution and statistical values. As a result, it was possible to grasp the damage situation on a mesh basis. In addition, it was possible to understand the removal status of the building, which shows the progress of restoration, on a polygon basis. From these results, we confirmed the effectiveness of texture analysis using high-resolution satellite images in grasping the damaged buildings before and immediately after the disaster and in the restoration situation 1 year after the disaster. In the future, we plan to apply the index value used for extracting bare ground to texture analysis and evaluate the effect. In addition, we will consider the improvement of accuracy by sorting out incorrect extraction factors and combining analysis methods.

\section{ACKNOWLEDGEMENTS}

The Quickbird and Worldview-3 images used in this study include copyrighted material of MAXAR, Digital Globe, Inc., All Rights Reserved. The GIS data used in this study, the perimeter of the building is a product of GSI (The Geospatial Information Authority of Japan). The building damage survey results are data published by the Architectural Institute of Japan (AIJ).

\section{REFERENCES}

Cabinet Office of Japan (CAO), 2017: Summary of damage situation in the Kumamoto earthquake sequence.2017. http://www.bousai. go.jp/updates/h280414jishin/index.html, (1 September 2017, in Japanese)

Chen, J., Chen, W., Dou, A., Li, W., Sun, Y.: Research on optimal observation scale for damaged Buildings after earthquake based on optimal feature space, Int. Arch. Photogramm. Remote Sens. Spatial Inf. Sci., XLII-3, 155-160, https://doi.org/10.5194/isprs-archives-XLII-3-155-2018, 2018.

Geospatial Information Authority of Japan (GSI), 2017: Inform ation about the 2016 Kumamoto earthquake, https://www.gsi.go. jp/BOUSAI/H27-kumamoto-earthquake-index.html, (1 April 20 17 , in Japanese)

Ghaffarian, S., Kerle, N.: Towards post-disaster debris identification for precise damage and recovery assessments from UAV and satellite Images, Int. Arch. Photogramm. Remote Sens. Spatial Inf. Sci., XLII-2/W13, 297-302, https://doi.org/10.5194/isprs-archives-XLII-2-W13-297-2019, 2019.

Haralick, R. M., Shanmugam, K., et al.: Textural features for im age classification, IEEE Transactions on systems, man, and cyb ernetics, No.6, 610-621, https://doi.org/10.1109/TSMC.1973.4 309314, 1973.

Hussain, E., Ural, S., Kim, K., Fu, C., Shan, J.: Building extraction and rubble mapping for city Port-au-Prince post-2010 earthquake with GeoEye-1 imagery and Lidar Data, Photogramm. Eng. Rem. S., 77, 1011-1023, DOI: 10.14358/PERS.77.10.1011, 2011.
Huyck, C. K., Adams, B. J., Cho, S., Chung, H.-C. Eguchi, R. T.: Towards Rapid Citywide Damage Mapping Using Neighborhood Edge Dissimilarities in Very High-Resolution Optical Satellite Imagery-Application to the 2003 Bam, Iran, Earthquake-, Earthquake Spectra, Vol.21, No.S1, S255-S266, DOI: 10.1193/1.2101907, 2005.

Miura, H., Midorikawa, S., Soh, C.: Texture Analysis of HighResolution Satellite Images for Damage Detection in the 2010 Haiti Earthquake, Journal of Japan Association for Earthquake Engineering, 2012, Vol.12, No.6, 6 2-6_20, https://doi.org/10.5610/jaee.12.6_2, 2012.

Naito, S., Tomozawa, H., Mori, Y., Nakamura, H., Fujiwara, H.: Building damage detection of the kumamoto earthquake utilizing image analyzing methods with aerial photographs, Journal of Disaster Research., Vol.13. No.5, 928-942, https://doi.org/10.20965/jdr.2018.p0928, 2018.

National Institute for Land and Infrastructure Management (NLIM) and Building Research Institute (BRI), 2017: A report to analyze the cause of damage to buildings in the Kumamoto earthquake, Technical Note of NILIM , No.997, http://www.nilim.go.jp/lab/bcg/siryou/tnn/tnn0997pdf/ks0997.p df, (13 August 2019, in Japanese)

Okada, S., Takai, N.: Classifications of structural types and damage patterns of buildings for earthquake field investigation. Journal of Structural and Construction Engineering (Transactions of AIJ), Vol.64, No.524, 65-72, https://doi.org/10.3130/aijs.64.65_5,1999.

Sumida, K., Isoda, H., Inoue, R., Mori, T., Tanaka, K., Sato, T.: Survey of Construction Situation in Mashiki Town Two Years After the 2016 Kumamoto Earthquakes, Journal of Japan Association for Earthquake Engineering, Vol.19, No.1, 1_211_33, https://doi.org/10.5610/jaee.19.1_21, 2019.

Suzuki, K., Liu, W., Matsuoka, M., Yamazaki, F.: Development of Building Inventory Data for Earthquake Damage Assessment in Tacna, Peru Using Satellite Imagery, Proceedings of the Japan association for earthquake engineering, Vol.16, No.3, 3_137-3_146, https://doi.org/10.5610/jaee.16.3_137, 2016.

Yamada, M., Ohmura, J. Goto, H.: Wooden Building Damage Analysis in Mashiki Town for the 2016 Kumamoto Earthquakes on April 14 and 16, Earthquake Spectra, 33, 1555-1572, https://doi.org/10.1193/090816eqs144m, 2017. 